Abstract

\title{
Acceleration and Maximum Running Phases in 60-m Sprint and Vertical Jump Performance ${ }^{\dagger}$
}

\author{
Ilias Ntoumas *, Giorgos Nounos, Pavlos Ioannidis and Vasileios Voutselas \\ School of Physical Education and Sport Science, University of Thessaly, 42100 Trikala, Greece \\ * Correspondence: iliasdoumas@gmail.com \\ + Presented at the 9th Greek Conference of Biochemistry and Physiology of Exercise, Thessaloniki, Greece, \\ 18-20 October 2019.
}

Published: 2 September 2019

\begin{abstract}
AIM: The purpose of the present study was to investigate the correlation between acceleration and maximum running phase in 60-m sprint and vertical jump performance. Furthermore, to investigate the factors that affect the acceleration phase, maximum running phase, and overall 60-m sprint performance. MATERIAL \& METHOD: Participants were 25 young amateur athletes, aged $18 \pm 1$ years, with body mass $64.64 \pm 13.39 \mathrm{~kg}$ and body height $1.71 \pm 0.11 \mathrm{~m}$. We examined the correlation between the acceleration phase $(0-30 \mathrm{~m})$ and maximum running phase (30-60 m) and 60-m sprint performance, measured with photocells (Optojump), and vertical jump performance (take-off velocity, jump height, maximal velocity, maximal power), measured with a force plate (Bertec). Pearson correlation was used to examine the correlation between the forementioned parameters (SPSS, v. 21). RESULTS: There was a correlation between jump height and 60-m sprint performance $(r=-0.713, p<0.001)$, maximum running phase $(r=-0.512, p=0.15)$, and a weak correlation with acceleration phase $(r=-0.495, p=0.19)$. There was a correlation between take-off velocity and 60-m sprint performance $(r=-0.732, p<0.001)$, maximum running phase $(r=$ $-0.553, p=0.08)$, and a weak correlation with acceleration phase $(r=-0.472, p=0.27)$. There was a weak correlation between maximal velocity, acceleration phase $(r=0.439, p=0.41)$, and 60-m sprint performance $(r=0.438, p=0.42)$. There was a correlation between maximal power and $60-\mathrm{m}$ sprint performance $(r=-0.739, p<0.001)$, acceleration phase $(p=-0.635, p=0.02)$, and a weak correlation with maximum running phase $(r=-0.437, p=0.042)$. There was a correlation between 60 -m sprint performance and maximum running phase $(r=0.792, p<0.001)$ and acceleration phase $(r=0.596, p$ $=0.03)$. Finally, there was a correlation between body height and 60-m sprint performance $(r=$ $-0.738, p=0.02)$ and maximum running phase $(r=-0.666, p=0.07)$. CONCLUSIONS: According to our results, 60-m sprint performance was highly correlated with body height, maximum running phase and all the vertical jump parameters (explosive power), except for maximal power, which had a high correlation with the acceleration phase (sprint acceleration).
\end{abstract}

Keywords: acceleration; sprint; vertical jump

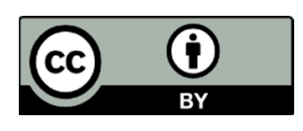

(C) 2019 by the authors. Submitted for possible open access publication under the terms and conditions of the Creative Commons Attribution (CC BY) license (http://creativecommons.org/licenses/by/4.0/). 